during 1, 2, 3, 9 or 18 breaths, with only minor and occasional side effects.

Original article Voswinckel R et al. (2006) Favorable effects of inhaled treprostinil in severe pulmonary hypertension: results from randomized controlled pilot studies. J Am Coll Cardiol 48: 1672-1681

\section{Cardiac surgery outcomes for Jehovah's Witnesses}

The religious beliefs of Jehovah's Witnesses prevent them from accepting blood transfusions or any blood-derived products; surgical protocols have, therefore, been adapted for these individuals. The safety of modified cardiac procedures for such patients has been previously demonstrated, but to date the outcomes of open heart surgery in Jehovah's Witnesses have not been compared directly with those of non-Jehovah's Witnesses.

Stamou et al. identified 49 adult Jehovah's Witnesses and 196 adult non-Jehovah's Witnesses who underwent comparable types of heart surgery at Inova Fairfax Hospital and Washington Hospital Center between January 1990 and July 2004. Surgical techniques, anesthesia, extracorporeal circulation and myocardial protection methods were the same for all patients, but Jehovah's Witnesses were maintained on a continuous blood circuit via a Cell Saver ${ }^{\circledR}$ (Haemonetics Corporation, Braintree, MA) washed blood bag. All blood left in the circuit was returned to the patient following surgery.

There were no significant differences between the groups with regard to the number of deaths during surgery, the duration of postoperative intensive care, or the total length of hospitalization following surgery, even after adjustment for gender, age, case priority, preoperative ejection fraction and coronary bypass surgery. The incidences of a range of short-term postoperative outcomes, including stroke, new-onset atrial fibrillation and acute myocardial infarction, were also similar.

As there was no long-term follow-up in this study, the enduring efficacy of successful open heart surgery in Jehovah's Witnesses remains to be proved. Nevertheless, short-term outcomes do not seem to be negatively affected by a patient choosing not to receive blood products.

Original article Stamou SC et al. (2006) Comparisons of cardiac surgery outcomes in Jehovah's versus non-Jehovah's Witnesses. Am J Cardiol 98: 1223-1225

\section{Incident statin use reduces the risk of death in patients with heart failure}

The use of statins in patients with heart failure is currently a matter of some debate. In an observational study, Go et al. demonstrate an association between the initiation of statin therapy and reductions in the risks of death and hospitalization in such patients.

Over a median period of 2.4 years, the investigators recorded initiation of statin therapy and incidence of all-cause death and hospitalization for heart failure in a population of 24,598 heart failure patients with no history of statin use. Those initiating statin therapy during follow-up $(n=12,648)$ were more likely to be younger and male, and had a higher prevalence of known coronary heart disease, diabetes and hypertension, than statin nonusers. During follow-up, 8,235 patients died and 9,215 patients were hospitalized for heart failure. When adjusted for age and sex, the mortality and hospitalization rates were lower in patients who initiated statin therapy than in those who did not (14.5 per 100 person-years vs 25.3 per 100 personyears, $P<0.001$, and 21.9 per 100 person-years vs 31.1 per 100 person-years, $P<0.001$, respectively). Multivariate analysis, using an intent-totreat approach, confirmed that incident statin use was associated with lower relative risks of death (adjusted hazard ratio $0.76,95 \% \mathrm{Cl}$ 0.72-0.80) and hospitalization (adjusted hazard ratio $0.79,95 \% \mathrm{Cl} 0.74-0.85)$, even following adjustment for sociodemographic characteristics, long-term use of other therapies and propensity to take a statin. The authors call for randomized controlled trials among patients with nonischemic heart failure to elucidate the role of statins in the heart failure setting.

Original article Go AS et al. (2006) Statin therapy and risks for death and hospitalization in chronic heart failure. JAMA 296: $2105-2111$

\section{Drug therapy combined with an LVAD can reverse heart failure}

Use of a left ventricular assist device (LVAD) can reverse left ventricular remodeling in patients with heart failure, but recovery sufficient to permit explantation has so far been achieved in only a small number of patients. To increase the recovery rate, researchers in the 